\title{
FRIGOCONSERVAÇÃO E CONTROLE DE PODRIDÕES EM LARANJA 'VALÊNCIA'
}

\author{
COLD STORAGE AND ROT CONTROL ON 'VALENCIA' ORANGE
}

\author{
Auri Brackmann $^{1}$ Rosangela Lunardi ${ }^{2}$ Joel Donazzolo ${ }^{3}$
}

\section{RESUMO}

Com o objetivo de estabelecer condições de armazenamento para a laranja 'Valência', foi conduzido um experimento avaliando o efeito de temperatura, condição de atmosfera controlada e aplicação pós-colheita de produtos químicos sobre a qualidade dos frutos e ocorrência de podridões. Foram avaliados os seguintes tratamentos: $0,5^{\circ} \mathrm{C}$ em armazenamento refrigerado $(A R) ; 3^{\circ} \mathrm{C}$ em $A R ; 3^{\circ} \mathrm{C}$ em AR e tratamento com Iprodione $(0,15 \%$ em imersão): $3^{\circ} \mathrm{C}$ em AR e tratamento com detergente $(2 \%$ em imersão); $3^{\circ} \mathrm{C}$ em atmosfera controlada (AC) com ar e $5 \%$ de $\mathrm{CO}_{2} ; 3^{\circ} \mathrm{C}$ em $\mathrm{AC}$ com $10 \%$ de $\mathrm{O}_{2}$ e $5 \%$ de $\mathrm{CO}_{2} ;$ e, $7^{\circ} \mathrm{C}$ em $\mathrm{AR}$. A umidade relativa do ar (UR) foi mantida entre 90 e 94\%. As avaliações foram realizadas aos 60 e aos 84 dias de armazenamento. Os parâmetros avaliados foram: perda de peso, acidez titulável, sólidos solúveis totais (SST) e podridões. Aos 60 dias, não houve diferença significativa entre os tratamentos com relação à acidez e aos $S S T$. A $3^{\circ} \mathrm{C}$ e $0,5^{\circ} \mathrm{C}$ houve menor perda de peso que na temperatura de $7^{\circ} \mathrm{C}$. Na avaliação aos 84 dias, os frutos armazenados em $A R$ a $7^{\circ} \mathrm{C}$ e $0,5^{\circ} \mathrm{C}$ apresentaram perda de peso superior aos demais tratamentos a $3^{\circ} \mathrm{C}$. A acidez e os SST diminuíram mais a $7^{\circ} \mathrm{C}$ em $A R$, porém não diferindo com $3^{\circ} \mathrm{C}$ em AR. A incidência de podridões, na segunda avaliação, não apresentou diferença estatística entre os frutos tratados e não tratados. A $7^{\circ} \mathrm{C}$ em AR houve maior incidência de podridões que a $3^{\circ} \mathrm{C}$ em $A R$, sendo que, em $0,5^{\circ} \mathrm{C}$ em $A R$, houve grande incidência de podridões, devido ao dano por baixa temperatura. Em AC houve também grande incidência de podridões decorrente de danos por altas concentrações de $\mathrm{CO}_{2}$ e baixas concentrações de $\mathrm{O}_{2}$.

Palavras-chave: armazenamento, podridões, fungicida, laranjas

\section{SUMMARY}

The experiment was carried out to establish storage conditions for 'Valência' orange, evaluating the effect of storage temperature, controlled atmosphere (CA) conditions and postharvest application of chemicals on the fruit quality and rot incidence. The treatments were: cold storage at $0.5^{\circ} \mathrm{C}$; cold storage at $3^{\circ} \mathrm{C}$; cold storage at $3^{\circ} \mathrm{C}$ and Iprodione treatment $\left(0.15 \%\right.$ deeping); cold storage at $3^{\circ} \mathrm{C}$ and detergent treatment (2\% deeping); $\mathrm{CA} 3^{\circ} \mathrm{C}$ with Air and $5 \%$ of $\mathrm{CO}_{2}$; $\mathrm{CA}$ with $10 \%$ of $\mathrm{O}_{2}$ and $5 \%$ of $\mathrm{CO}_{2}$ at $3{ }^{\circ} \mathrm{C}$; and cold storage at $7^{\circ} \mathrm{C}$. Relative humidity (RH) was maintained between 90 and 94\%. The evaluations were preformed after 60 and 84 storage days. After 60 storage days there was no statistic difference among treatments on the titratable acidity and total soluble solids (TSS). At $3^{\circ} \mathrm{C}$ and $0.5^{\circ} \mathrm{C}$ there was a lower weight loss than at $7^{\circ} \mathrm{C}$. After 84 storage days the fruits in cold storage at 7 and $0.5^{\circ} \mathrm{C}$ showed higher weight loss than at $3^{\circ} \mathrm{C}$. The acidity and TSS were lowest for cold storage at $7^{\circ} \mathrm{C}$, but there was no difference with $3^{\circ} \mathrm{C}$. The rot incidence, in the second evaluation, Iprodione application and the detergent had no difference with the oranges without chemicals. In cold storage at $7^{\circ} \mathrm{C}$ there was higher rot incidence than at $3^{\circ} \mathrm{C}$. At $0.5^{\circ} \mathrm{C}$ there was high rot incidence due to low temperature damage. At CA condition there was high rot incidence due to high $\mathrm{CO}_{2}$ and low $\mathrm{O}_{2}$ damage.

Key words: storage, rot, fungicid, orange.

\section{INTRODUÇÃO}

O Brasil destaca-se no cenário mundial como o primeiro produtor de laranjas e o principal exportador de suco concentrado (FIORAVANÇO \& MÂNICA, 1994). O controle de podridões póscolheita é um dos aspectos de maior importância no mercado de citros destinados ao consumo in natura (GUERRA et al., 1990) e, para prolongar o período de oferta da laranja 'Valência', torna-se necessário o armazenamento refrigerado.

\footnotetext{
${ }^{1}$ Engenheiro Agrônomo, Doutor, Professor Adjunto, Departamento de Fitotecnia, Centro de Ciências Rurais (CCR), Universidade Federal de Santa Maria (UFSM), 97105-900, Santa Maria, RS. E-mail: fruti@ super.ufsm.br. Autor para correspondência.

${ }^{2}$ Engenheiro Agronômo, aluna do Curso de Pós-graduação em Agronomia, Universidade Federal do Rio Grande do Sul, Porto Alegre, RS.

${ }^{3}$ Engenheiro Agrônomo, extensionista da EMATER/RS. 
A temperatura, isoladamente, é o fator mais importante que afeta a manutenção da qualidade de uma fruta armazenada, porque os processos metabólicos são dependentes da temperatura dentro de uma faixa fisiológica ideal. As frutas cítricas demonstram sensibilidade a baixas temperaturas, que se manifesta através da morte de células da casca ("pitting"), formação de manchas circulares e deprimidas de coloração marrom, escurecimento da polpa e alterações do gosto e sabor (FIORAVANÇO \& MÂNICA, 1994). De acordo com Chalutz, apud COHEN (1990), o armazenamento de citros a $12^{\circ} \mathrm{C}$ não causa dano pelo frio ("chilling") e a incidência de podridões é relativamente baixa, porém, a $6^{\circ} \mathrm{C}$ os frutos apresentam "pitting" e, subseqüentemente, desenvolvem-se podridões nos frutos danificados (15 a 70\% após doze semanas). CHITARRA \& CHITARRA (1990) recomendam temperatura de armazenamento, para a laranja 'Valência', de 6,1 a $9,4^{\circ} \mathrm{C}$ com uma umidade relativa de 88 a $92 \%$, para um período de armazenamento de cinco a seis meses. Segundo HARDENBURG et al. (1986), na Flórida e Texas, a laranja 'Valência' pode ser armazenada por oito a doze semanas de 0 a $1^{\circ} \mathrm{C}$, enquanto na Califórnia e Arizona, em $3^{\circ} \mathrm{C}$, ela pode se conservar por seis a oito semanas ou, em 5 a $7^{\circ} \mathrm{C}$, por quatro a seis semanas, ou ainda em $9^{\circ} \mathrm{C}$, por três a quatro semanas. Mas, conforme DÜNDAR \& PECKMEZCI (1990), a temperatura mais adequada para a laranja 'Valência' é $4^{\circ} \mathrm{C}$ para um armazenamento de cinco meses, sem haver perda significativa de qualidade.

\section{DONAZZOLO \& BRACKMANN} (1995), trabalhando com laranja de umbigo cv. Monte Parnaso, observaram que a melhor temperatura de armazenamento é a $3^{\circ} \mathrm{C}$, com umidade de $97 \%$, podendo ser armazenada por um período de até dois meses.

O armazenamento em atmosfera controlada (AC) para prolongar o período de conservação de laranjas ainda tem sido pouco usado (HARDENBURG $\boldsymbol{e} \boldsymbol{t} \boldsymbol{a l} .$, 1986). Na Flórida, laranjas 'Valência' armazenadas em AC com concentrações de $15 \%$ de $\mathrm{O}_{2}$ e 0 ou $5 \%$ de $\mathrm{CO}_{2}$ por doze semanas a $1{ }^{\circ} \mathrm{C}$, seguido por uma semana em $21^{\circ} \mathrm{C}$, resultaram em frutos com poucas perdas de sabor e poucos danos na casca, embora não tenha sido eficiente no controle de podridões (HARDENBURG et al., 1986). Já CHITARRA \& CHITARRA (1990) citam que, para laranjas, as melhores concentrações de $\mathrm{O}_{2}$ e $\mathrm{CO}_{2}$ são 10 e $5 \%$, respectivamente, na temperatura de 5 a $10^{\circ} \mathrm{C}$. KE \& KADER (1990) testaram $60 \%$ de $\mathrm{CO}_{2}$ na temperatura de $5^{\circ} \mathrm{C}$ por cinco a quatorze dias, seguidos por armazenamento refrigerado a $5^{\circ} \mathrm{C}$ por sete dias e a laranja 'Valência' apresentou somente leve escurecimento na casca.
As podridões representam um dos maiores problemas na limitação da vida pós-colheita de frutas cítricas (KADER, 1985). Segundo COHEN (1990), a temperatura mínima para o desenvolvimento de muitos fungos in vitro é entre 4 a $5^{\circ} \mathrm{C}$. GUERRA et al. (1990) afirmam que frutas cítricas não tratadas com fungicidas podem desenvolver de 25 a $50 \%$ de podridões, quando são oferecidas ótimas condições para o desenvolvimento dos fungos.

O objetivo deste trabalho foi de avaliar o efeito das temperaturas em armazenamento refrigerado (AR) e condições de armazenamento em atmosfera controlada (AC), associado à aplicação de produtos químicos em pós-colheita, sobre a qualidade da laranja 'Valência' e, em especial, na ocorrência de distúrbios fisiológicos e podridões.

\section{MATERIAL E MÉTODOS}

O experimento foi conduzido no Núcleo de Pesquisa em Pós-colheita (NPP) do Departamento de Fitotecnia da Universidade Federal de Santa Maria, no período de outubro de 1994 a janeiro de 1995, com laranjas da cv. Valência, provenientes de um pomar comercial de Cachoeira do Sul RS.

O delineamento experimental foi inteiramente casualizado com duas repetições de 75 frutos. Os tratamentos foram combinações de temperatura e condições de atmosfera: $0,5^{\circ} \mathrm{C}$ em armazenamento refrigerado (AR); $3^{\circ} \mathrm{C}$ em $\mathrm{AR} ; 3^{\circ} \mathrm{C}$ em $\mathrm{AR}$ e tratamento com Iprodione $\left(0,15 \%\right.$ em imersão); $3^{\circ} \mathrm{C}$ em AR e tratamento com detergente marca 'Odd' (2\% em imersão); $3^{\circ} \mathrm{C}$ em atmosfera controlada (AC) com $\operatorname{ar} / 5 \% \mathrm{CO}_{2} ; 3^{\circ} \mathrm{C}$ em $\mathrm{AC}$ com $10 \% \mathrm{O}_{2} / 5 \% \mathrm{CO}_{2}$; e, $7^{\circ} \mathrm{C}$ em AR. A umidade relativa (UR) foi mantida entre 90 e $94 \%$.

Após seleção dos frutos e homogeneização das amostras, estas foram armazenadas em câmaras experimentais com volume de 232 litros, que continham dois orifícios de $0,5 \mathrm{~cm}$ de diâmetro para permitir as trocas de gases para os frutos em AR, e para os que permaneceram em AC, a câmara ficou hermeticamente fechada. A aplicação dos tratamentos com Iprodione $(0,15 \%)$ e detergente marca 'Odd' ( $2 \%$ ) foi feita por imersão, em pós-colheita, durante dois minutos. A manutenção da concentração desejada de gases nas câmaras de AC foi obtida através da análise diária com uso de analisadores de $\mathrm{O}_{2}$ e $\mathrm{CO}_{2}$, marca Agri-Datalog e correção diária das concentrações, que variavam em função da respiração dos frutos. Os níveis de gás carbônico foram mantidos através da absorção do $\mathrm{CO}_{2}$ com a circulação do gás de cada câmara por um absorvedor de $\mathrm{CO}_{2}$, contendo hidróxido de potássio. Para compensar o oxigênio consumido pelos frutos, foi injetado, diariamente, ar nas câmaras. 
As avaliações da qualidade dos frutos foram realizadas na instalação do experimento e aos 60 e 84 dias de armazenamento. As variáveis analisadas foram:

a) Sólidos Solúveis Totais (SST) - determinados a partir do suco extraído das amostras de frutos, com um refratômetro manual, e os resultados foram expressos em graus Brix;

b) acidez titulável - determinada pela titulação de $10 \mathrm{ml}$ de suco, da mesma amostra utilizada para determinação dos SST, diluídos em 100ml de água destilada, com uma solução de $\mathrm{NaOH} 0,1 \mathrm{~N}$ até $\mathrm{pH}$ 8,1 , e os resultados expressos em $\mathrm{cmol}_{\mathrm{c}} . \mathrm{L}^{-1}$;

c) podridões - obtidas pela percentagem de frutos que apresentavam lesões com diâmetro maior que $0,5 \mathrm{~cm}$, com características típicas de ataque de fungos;

d) perda de peso - determinada pela diferença de peso dos frutos na instalação do experimento e após o armazenamento;

e) análise sensorial - feita por um grupo de quatro pessoas que, degustando os frutos, avaliaram a existência de sabor e aroma estranhos.

Os dados foram submetidos à análise de variância e ao teste de Duncan em nível de 5\% de probabilidade de erro. As variáveis perda de peso e podridões, expressas em percentagem, foram transformadas em arcoseno antes da análise de variância.

\section{RESULTADOS E DISCUSSÃO}

Após 60 dias de armazenamento, não houve diferença estatística entre os tratamentos com relação à acidez e SST (tabela 1), tanto em condições de AC como em AR. Na avaliação aos 84 dias (tabela 2), os SST e acidez titulável tenderam a se manter mais elevados nas temperaturas de 0,5 e $3^{\circ} \mathrm{C}$, sem diferir estatisticamente do tratamento com temperatura de $7^{\circ} \mathrm{C}$. Nas condições de armazenamento em $\mathrm{AC}$ a $3^{\circ} \mathrm{C}$ em ar e $5 \%$ de $\mathrm{CO}_{2}$ e $\mathrm{AR}$ a $7^{\circ} \mathrm{C}$, a acidez titulável e os SST diferiram entre si, onde em AC os frutos apresentaram melhor qualidade, com níveis superiores de acidez titulável e SST. Normalmente, altos teores de açúcares e acidez estão associados à melhor qualidade dos frutos e ao maior potencial de armazenamento. As frutas cítricas, após a colheita, continuam a acumular SST devido à conversão de ácidos orgânicos em glicolíticos intermediários e, subseqüentemente, a hexoses. De modo geral, o conteúdo de ácidos orgânicos nas frutas aumenta durante os primeiros estádios de desenvolvimento e, em seguida, decresce lenta e progressivamente durante e após o processo de maturação (FIORAVANÇO \& MÂNICA, 1994). Porém, pelas tabelas 1 e 2, observa-se que houve um acréscimo da acidez com aumento do período de armazenamento, possivelmente devido ao acúmulo dos ácidos no interior da célula como resultado da desidratação do fruto. Comportamento semelhante de aumento da acidez foi observado por BRACKMANN \& DONAZZOLO (1996) com laranjas 'Monte Parnaso'.

A ocorrência de podridões, na primeira avaliação (tabela 1), teve as menores porcentagens na temperatura de $3^{\circ} \mathrm{C}$ em $\mathrm{AR}$, não havendo efeito

Tabela 1 - Qualidades físico-químicas de laranja 'Valência' após 60 dias de armazenamento refrigerado e em atmosfera controlada, Santa Maria, RS, 1995.

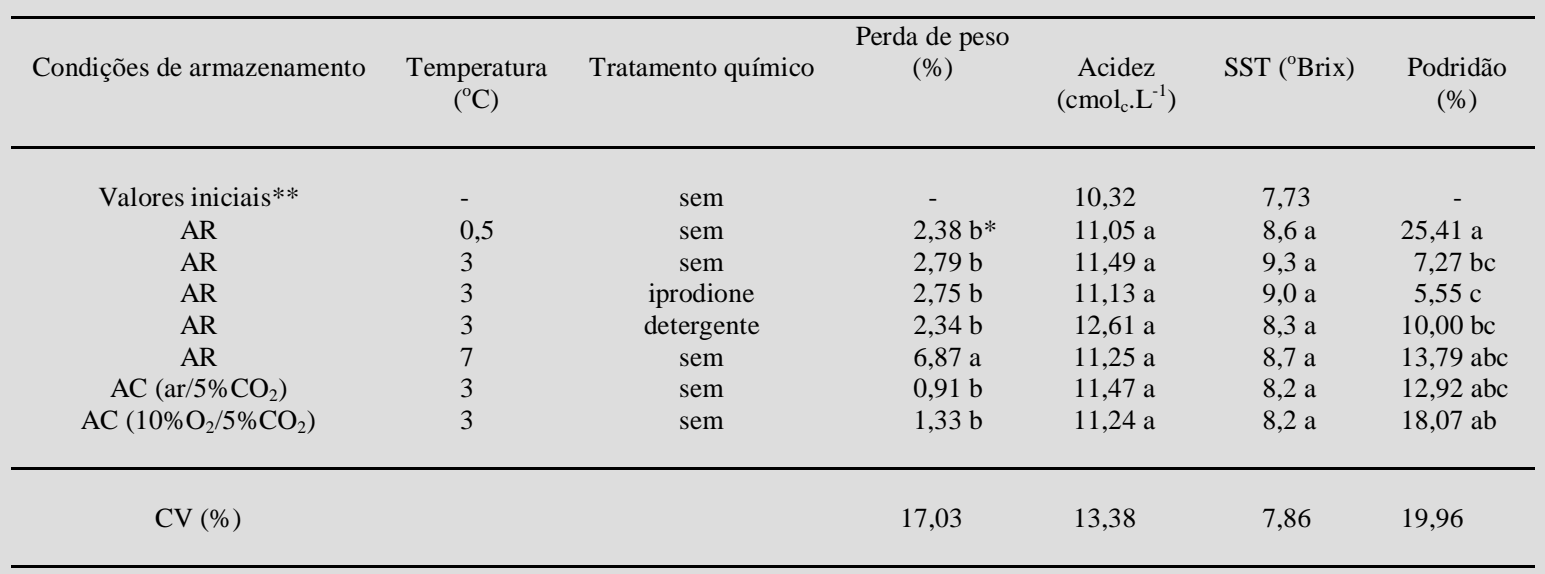

* Os valores não seguidos pela mesma letra na vertical, diferem entre si pelo teste de Duncan em nível de 5\% de probabilidade de erro.

** Análise fisico-química realizada na instalação do experimento.

$\mathrm{AR}=$ Armazenamento Refrigerado

$\mathrm{AC}=$ Atmosfera Controlada 
Tabela 2 - Qualidades físico-químicas de laranja 'Valência' após 84 dias de armazenamento refrigerado e em atmosfera controlada, Santa Maria, RS, 1995

\begin{tabular}{|c|c|c|c|c|c|c|}
\hline Condições de armazenamento & $\begin{array}{c}\text { Temperatura } \\
\left({ }^{\circ} \mathrm{C}\right)\end{array}$ & Tratamento químico & $\begin{array}{c}\text { Perda de peso } \\
(\%)\end{array}$ & $\begin{array}{c}\text { Acidez } \\
\left(\mathrm{cmol}_{\mathrm{c}} \cdot \mathrm{L}^{-1}\right)\end{array}$ & SST ( ${ }^{\circ}$ Brix $)$ & $\begin{array}{c}\text { Podridão } \\
(\%)\end{array}$ \\
\hline $\mathrm{AR}$ & 0,5 & sem & $4,49 \mathrm{ab}^{*}$ & $12,68 \mathrm{ab}$ & $8,6 \mathrm{a}$ & $80,05 \mathrm{a}$ \\
\hline $\mathrm{AR}$ & 3 & sem & $3,28 \mathrm{ab}$ & $12,23 \mathrm{~b}$ & $8,3 \mathrm{ab}$ & $24,41 \mathrm{~cd}$ \\
\hline $\mathrm{AR}$ & 3 & iprodione & $2,69 \mathrm{bc}$ & $12,49 \mathrm{ab}$ & $8,5 \mathrm{ab}$ & $14,50 \mathrm{~d}$ \\
\hline $\mathrm{AR}$ & 3 & detergente & $2,25 \mathrm{bc}$ & $13,63 \mathrm{ab}$ & 8,6 a & $25,57 \mathrm{~cd}$ \\
\hline $\mathrm{AR}$ & 7 & sem & $7,15 \mathrm{a}$ & $12,17 \mathrm{~b}$ & $8,2 \mathrm{~b}$ & $36,78 \mathrm{bc}$ \\
\hline $\mathrm{AC}\left(\operatorname{ar} / 5 \% \mathrm{CO}_{2}\right)$ & 3 & sem & $1,93 \mathrm{bc}$ & 13,96 a & $8,6 \mathrm{a}$ & $47,36 \mathrm{~b}$ \\
\hline $\mathrm{AC}\left(10 \% \mathrm{O}_{2} / 5 \% \mathrm{CO}_{2}\right)$ & 3 & sem & $0,63 \mathrm{c}$ & $13,19 \mathrm{ab}$ & $8,5 \mathrm{ab}$ & 63,31 a \\
\hline $\mathrm{CV}(\%)$ & & & 17,10 & 5,06 & 1,67 & 11,53 \\
\hline
\end{tabular}

estatístico significativo dos tratamentos com Iprodione e detergente. Nas condições de $\mathrm{AC}$ a $3^{\circ} \mathrm{C}(10 \%$ de $\mathrm{O}_{2}$ e $5 \%$ de $\mathrm{CO}_{2}$ ), a porcentagem de podridão foi maior que em $\mathrm{AR}$ a $3^{\circ} \mathrm{C}$, sem tratamento químico. Porém, na temperatura de $0,5^{\circ} \mathrm{C}$ ocorreu a maior porcentagem de frutos podres, possivelmente devido ao dano por baixa temperatura, a qual poderia ter provocado danos na epiderme dos frutos, predispondo-os ao ataque de patógenos. Já na segunda avaliação (tabela 2), a maior ocorrência de podridão continuou sendo na temperatura de $0,5^{\circ} \mathrm{C}$, não diferindo estatisticamente da condição de armazenamento em AC na concentração de $10 \% \mathrm{O}_{2}$ e $5 \% \mathrm{CO}_{2}$. A menor porcentagem de frutos podres ocorreu na temperatura de $3^{\circ} \mathrm{C}$ com uso de Iprodione, embora não tenha havido efeito significativo comparado à aplicação de detergente, nessa temperatura. No armazenamento em AC houve grande incidência de podridões, confirmando os resultados de HARDENBURG et $\boldsymbol{a l}$. (1986), os quais afirmaram que as condições de AC não apresentam efeito sobre o controle de podridões. É provável que a redução da concentração de $\mathrm{O}_{2} \mathrm{e} o$ aumento do $\mathrm{CO}_{2}$ tenham causado danos fisiológicos às células, predispondo-as ao ataque de fungos.

O percentual de perda de peso na primeira e segunda avaliações (tabelas 1 e 2) foi maior na temperatura de $7^{\circ} \mathrm{C}$ devido à grande desidratação decorrente de uma maior diferença entre as pressões de vapor do interior do fruto e o ambiente externo, e, também, devido à maior atividade metabólica dos frutos. Com relação às condições de armazenamento em AC, em ambas as avaliações, a percentagem de perda de peso foi menor, se comparado com os demais tratamentos, devido aos frutos respirarem me- nos. O uso de Iprodione ou detergente não interferiram na perda de peso.

\section{CONCLUSÕES}

A melhor temperatura para armazenamento da laranja 'Valência' é de $3^{\circ} \mathrm{C}$. O armazenamento refrigerado a $0,5^{\circ} \mathrm{C}$ aumenta a incidência de podridões, provavelmente devido a danos por baixa temperatura ("chilling"). O armazenamento em AC causa danos aos frutos, predispondo-os à ocorrência de podridões e a aplicação pós-colheita de Iprodione não diminui a ocorrência de podridões.

\section{REFERÊNCIAS BIBLIOGRÁFICAS}

BRACKMANN, A., DONAZZOLO, J. Armazenamento refrigerado de laranja cv. Monte Parnaso. Rev Bras Frutic, Cruz das Almas, v. 18, n. 3, p. 311-317, 1996.

CHITARRA, M.I.F., CHITARRA, A.B. Pós-colheita de frutos e hortaliças: fisiologia e manuseio. Lavras: ESAL-FAEPE, 1990. 293 p.

COHEN, E. The use of temperature for posthavest decay control in citrus fruit. In: BIOLOGICAL CONTROL OF POSTHARVEST DISEASES OF FRUIT AND VEGETABLES WORKSHOP, 1990. Shepherdstow. Proceedings... Shepherdstow, 1990, p. 256-267.

DONAZZOLO, J., BRACKMANN, A. Armazenamento refrigerado de laranja cv. Monte Parnaso. In: JORNADA INTEGRADA DE PESQUISA, EXTENSÃO E ENSINO, 2, 1995. Santa Maria, RS. Anais... Pró-Reitoria de Graduação e Pesquisa, 1995, Santa Maria, 1036 p., p. 416.

DÜNDAR, Ö., PECKMEZCI, M. Investigations on cold storage and postharvest physiology of Valencia and Konzan Yerli orange varieties. In: INTERNATIONAL HORTICULTURAL CONGRESS, 23, 1994, Firenze. Abstracts... Firenze, 1990, v. 1, p. 682 . 
FIORAVANÇO, J.C., MÂNICA, I. Armazenamento de frutas cítricas em temperatura controlada. Cadernos de Horticultura, Porto Alegre, v. 2, n. 2, p. 1-8, 1994.

GUERRA, E., OTERO, O., MOYA, C.B. Síntesis de los trabajos realizados para el control de las pudriciones poscosecha de los frutos cítricos en Cuba. Ciencia y Técnica en la Agricultura, Havana, v. 13, n. 3-4, p. 35-55, 1990.

HARDENBURG, R.E., WATADA, A.E., WANG, C.Y. The comercial storage of fruits, vegetables and florist and nursery stocks. Washington: U.S. Departament of Agriculture, 1986. 136p. Agriculture Handbook, 66.

KADER, A.A. Postharvest handling systems: Subtropical fruits. In: DIVISION OF AGRICULTURE AND NATURAL RESOURCES. Postharvest tecnology of horticultural crops. Califórnia, 1985, p. 152-156.

KE, D., KADER, A.A. Tolerance of 'Valencia' oranges to controlled atmospheres as determined by physiological responses and quality attributes. Journal of the American Society for Horticultural Science, Alexandria, v. 5, n. 115, p. $779-783,1990$.

Ciência Rural, v. 29, n. 2, 1999. 